\section{References}

1. Papanagnou DOS. Sea Snake Envenomation. (http:// emedicine.medscape.com/article/771804-overview)

2. Somaweera R, Somaweera N. An overview of Sri Lankan sea snakes with an annotated check list and a field key. Taprobanica 2009; 1: 43-54.

3. Senanayake MP, Ariaratnam CA, Abeywickrema S,
Belligaswatte A. Two Sri Lankan cases of identified sea snake bites, without envenoming. Toxicon 2005; 45: 861-3.

4. Fernando M, Gooneratne W. Sea-snake envenoming. Ceylon Medical Journal 1983; 28: 131-43.

5. Amarasekera N, Jayawardena A, Ariyaratnam A, Hewage UC, de Silva A. Bite of a sea snake (Hydrophis spiralis): a case report from Sri Lanka. The Journal of Tropical Medicine and Hygiene 1994; 97:195-8.

\title{
Bilateral psoas abscess in a renal transplant patient
}

\author{
A S Badurdeen ${ }^{1}$, P N Rajakrishna'1, H M N J Herath ${ }^{1}$, A W M Wazil', N Nanayakkara' \\ Ceylon Medical Journal 2012; 57: 175-176
}

\section{Introduction}

Psoas abscess is a rare complication of transplantation. There is often delay in diagnosis as its relative infrequency and the non specific signs and symptoms. We present a case of bilateral psoas abscessess developing two months after a living donor kidney transplant.

\section{Case report}

A 51-year old male with type 2 diabetes mellitus who underwent living donor kidney transplantation two months earlier was admitted with low back pain, general ill health, and a $5 \mathrm{~kg}$ weight loss over one month. Immediate post transplant period was complicated by an acute tubular necrosis of the graft and he was intermittently dialysed via a femoral line for ten days, as his shunt flow was inadequate. The post transplant course was otherwise uncomplicated, with allograft function returning to normal in two weeks. He was given cyclosporin, mycophenolate mofetil, and prednisolone. On examination, he had bilateral flank tenderness and a normal gait. Neurologically the lower limbs were normal. His oral temperature was $37.4^{\circ} \mathrm{C}$, blood pressure was 110/80 $\mathrm{mm} \mathrm{Hg}$ and pulse rate was 94/ $\min$.

He had a white blood cell count of $27 \times 10^{6} / \mu$ l with $83 \%$ neutrophils, an erythrocyte sedimentation rate of 97 $\mathrm{mm} /$ hour, a C-reactive protein level of $31.7 \mathrm{mg} / \mathrm{dl}$ and a serum creatinine level of $10^{9} \mu \mathrm{mol} / \mathrm{L}$. After the initial USSAbdomen, computed tomography imaging of his pelvis demonstrated bilateral psoas abscessess, left measuring $10 \mathrm{~cm} \times 7 \mathrm{~cm}$ and right $7 \mathrm{~cm} \times 5 \mathrm{~cm}$ (Figures 1 and 2) without any evidence of intervertebral disc involvement. The abscesses were drained initially under ultrasound guidance followed by open surgery due to poor response. Staphylococcus aureus was isolated from the pus culture. Anaerobic cultures and Mycobacterium tuberculosis were negative. Blood cultures were also negative. He was treated with intravenous meropenum cloxacillin. After six weeks of oral cloxacillin treatment erythrocyte sedimentation rate was $18 \mathrm{~mm} / \mathrm{hr}$ and C-reactive protein level was $1.7 \mathrm{mg} / \mathrm{dl}$. No relapses have occurred more than three months after completion of treatment.

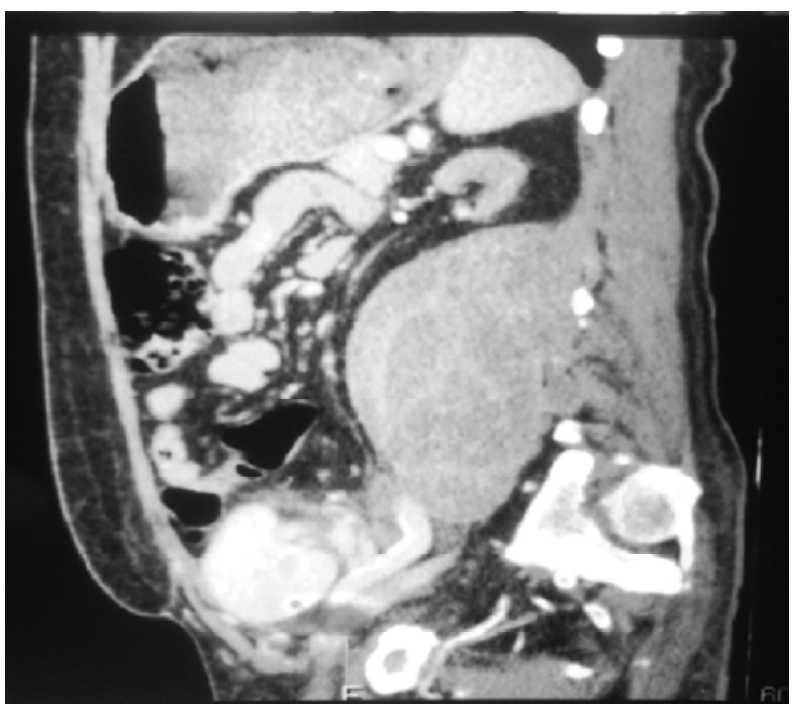

Figure 1. Coronal section of psoas abscess.

\section{${ }^{1}$ Teaching Hospital, Kandy, Sri Lanka.}

Correspondence: ASB, e-mail: <sadathba@gmail.com>. Received 12 March and revised version accepted 28 July 2012. Competing interests: none declared. 


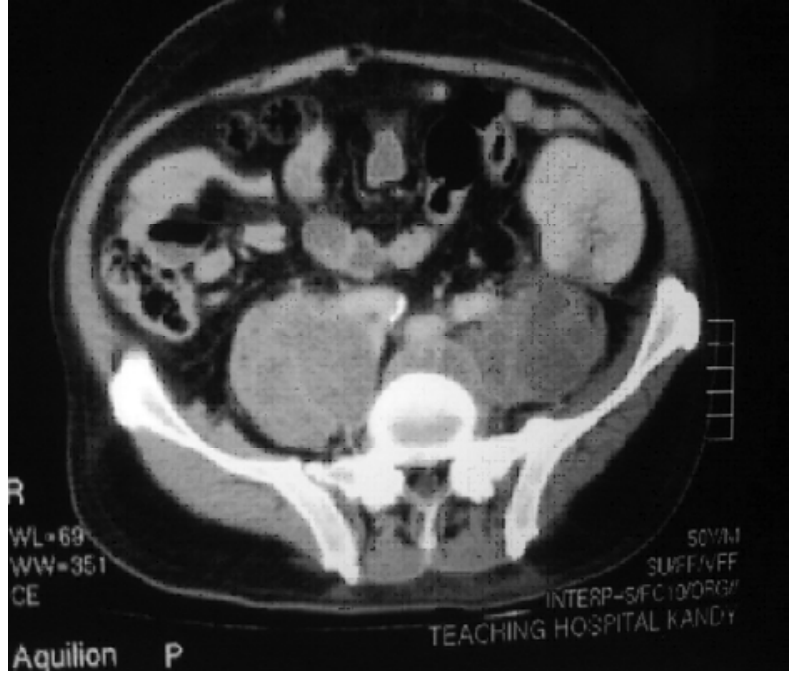

Figure 2. Cross section of psoas abscess with the transplanted kidney

\section{Discussion}

Psoas abscess is rare in post transplantation patients who receive immunosuppressive therapy. It can be classified as primary or secondary. It can occur in patients with diabetes mellitus, intravenous drug abuse, AIDS and after renal, heart and bone marrow transplantation. Primary psoas abscess occurs probably from the haematologic spread of organisms. Conditions associated with secondary psoas abscess include gastrointestinal diseases, genitourinary problems, femoral vessel catheterisation, vertebral osteomyelitis and endocarditis [2]. In this patient it is not clear whether this was a primary or secondary abscess as he had undergone a femoral vein catheterisation two months back. Although there were no overt signs of catheter infection, he may have had a bacteraemia.

In Asia and Africa, 99\% of psoas abscesses are primary, whereas in Europe and North America the incidence is lower; 17 and 61\%, respectively [2]. They are more common on the right side. $S$. aureus is implicated in over $88 \%$ of patients with primary psoas abscess. Other causative organisms include Streptococcus $s p$, Escherichia coli, Mycobacterium tuberculosis, Mycoplasma pneumoniae, Proteus, Pasteurella and Bacteroides spp [3]. A primary psoas abscess has a good prognosis, with a mortality rate of $2.4 \%$ compared to $18.9 \%$ for patients with a secondary abscess [4].

The classical clinical triad consisting of fever, back pain and limp is well known [5]. Our patient had a non specific presentation. A CT scan is considered the 'gold standard' for definitive diagnosis [2, 6]. Other laboratory findings include anaemia, an elevation of the white blood cell count, and increases in the $\mathrm{C}$-reactive protein level and erythrocyte sedimentation rate.

The treatment of a psoas abscess involves drainage of the abscess along with the use of appropriate antibiotics. Patients with a suspected primary psoas abscess should be treated with anti-Staphylococcal antibiotics as empirical treatment $[4,6]$. Drainage of the abscess should be performed through image-guided percutaneous drainage, which is less invasive, or surgical drainage, which is reported by some authors to be superior in achieving prompt recovery, as in our case [6].

\section{References}

1. Yang W-C, Lin S-F. Bone Marrow Transplantation 2006; 38: $73-4$.

2. Mallick IH, Thoufeeq MH, Rajendran TP. Iliopsoas abscesses. Postgraduate Medical Journal 2004; 80: 459-62.

3. O'Sullivan MV, Isbel NM, Johnson DW, et al. Disseminated pyogenic Mycoplasma pneumoniae infection in a renal transplant recipient, detected by broad-range polymerase chain reaction. Clinical Infectious Diseases 2004; 39: e98-9.

4. Katara AN, Shah RS, Bhandarkar DS, Unadkat RJ. Retroperitoneoscopic drainage of a psoas abscess. Journal of Pediatric Surgery 2004; 39: e4-e5.

5. Mynter H. Acute psoitis. Buffalo Medical and Surgical Journal 1881; 21: 202-10.

6. Santaella RO, Fishman EK, Lipsett PA. Primary vs secondary iliopsoas abscess. Prevention, microbiology, and treatment. Archives of Surgery 1995; 130: 1309-13. 